\title{
Improved Motion Perception and Impaired Spatial Suppression following Disruption of Cortical Area MT/V5
}

\author{
Duje Tadin, ${ }^{1}$ Juha Silvanto, ${ }^{2,3}$ Alvaro Pascual-Leone, ${ }^{2}$ and Lorella Battelli ${ }^{2,4}$ \\ ${ }^{1}$ Center for Visual Science and Department of Brain and Cognitive Sciences, University of Rochester, Rochester, New York 14627, ${ }^{2 B e r e n s o n-A l l e n ~ C e n t e r ~}$ \\ for Noninvasive Brain Stimulation and Department of Neurology, Beth Israel Deaconess Medical Center, Harvard Medical School, Boston, Massachusetts \\ 02215, ${ }^{3}$ Brain Research Unit, Low Temperature Laboratory and Advanced Magnetic Imaging Centre, Aalto University School of Science and Technology, \\ FI-00076 Aalto, Espoo, Finland, and ${ }^{4}$ Center for Neuroscience and Cognitive Systems, Italian Institute of Technology, 38068 Rovereto (TN), Italy
}

As stimulus size increases, motion direction of high-contrast patterns becomes increasingly harder to perceive. This counterintuitive behavioral result, termed "spatial suppression," is hypothesized to reflect center-surround antagonism—a receptive field property ubiquitous in sensory systems. Prior research proposed that spatial suppression of motion signals is a direct correlate of center-surround antagonism within cortical area MT. Here, we investigated whether human MT/V5 is indeed causally involved in spatial suppression of motion signals. The key assumption is that a disruption of neural mechanisms that play a critical role in spatial suppression could allow these normally suppressed motion signals to reach perceptual awareness. Thus, our hypothesis was that a disruption of MT/V5 should weaken spatial suppression and, consequently, improve motion perception of large, moving patterns. To disrupt MT/V5, we used offline $1 \mathrm{~Hz}$ transcranial magnetic stimulation (TMS) - a method that temporarily attenuates normal functioning of the targeted cortex. Early visual areas were also targeted as a control site. The results supported our hypotheses and showed that disruption of MT/V5 improved motion discrimination of large, moving stimuli, presumably by weakening surround suppression strength. This effect was specific to MT/V5 stimulation and contralaterally presented stimuli. Evidently, the critical neural constraints limiting motion perception of large, high-contrast stimuli involve MT/V5. Additionally, our findings mimic spatial suppression deficits that are observed in several patient populations and implicate impaired MT/V5 processes as likely neural correlates for the reported perceptual abnormalities in the elderly, patients with schizophrenia and those with a history of depression.

\section{Introduction}

Center-surround antagonism is a simple, yet powerful receptive field property that is found across sensory modalities, ranging from vision to electroperception (Tadin and Lappin, 2005a). In vision, center-surround antagonism is typically manifested as the reduction of a neuron's firing when the preferred stimulus is increased in size to include the inhibitory surround region. Consequently, such neurons respond poorly to large, uniform stimuli. Theoretical and neurophysiological work associates this basic mechanism with key neural processes, including redundancy reduction, input normalization, figure-ground segregation and computation of object motion (Born et al., 2000; Vinje and Gallant, 2000; Schwartz and Simoncelli, 2001; Pack et al., 2005).

\footnotetext{
Received June 26, 2010; revised 0ct. 18, 2010; accepted Nov. 18, 2010.

This work was supported by National Eye Institute (NEI) Grant R01 EY019295 to D.T., the "Harvard Catalyst," and the Harvard-Thorndike Clinical Research Center at Beth Israel Deaconess Medical Center [National Center for Research Resources (NCRR)-National Institutes of Health (NIH) Grant UL1 RR025758]. A.P.-L. was supported in part by NCRR-NIH Grant K24 RR018875 and NEI-NIH Grant R01 EY12091. L.B. was supported by NIH Grant R01-DC006842. We thank John Assad, Christopher Pack, and Joel Pearson for helpful comments on the manuscript, and Davis Glasser and Zaira Cattaneo for their help in conducting experiments. The content is solely the responsibility of the authors and does not necessarily represent the official views of the National Institutes of Health.

Correspondence should be addressed to Duje Tadin, 317 Meliora Hall, Department of Brain and Cognitive Sciences, University of Rochester, Rochester, NY 14627. E-mail: duje@cvs.rochester.edu.

DOI:10.1523/JNEUROSCI.4121-10.2011

Copyright $\odot 2011$ the authors $\quad 0270-6474 / 11 / 311279-05 \$ 15.00 / 0$
}

Despite their omnipresence at the neural level, we have relatively limited evidence linking different types of center-surround antagonism with their immediate behavioral correlates in human subjects. In motion perception, it has been proposed that spatial suppression - a counterintuitive elevation of direction discrimination thresholds with increasing stimulus size-is a direct perceptual correlate of center-surround antagonism within cortical area MT/V5 (Tadin et al., 2003). This linking hypothesis is supported by several studies offering largely correlational evidence. For example, the dependency of spatial suppression on stimulus size and eccentricity matches that of center-surround MT/V5 neurons (Tadin et al., 2003). Additionally, the finding that spatial suppression strength decreases at low contrast (Tadin et al., 2003) foreshadowed the discovery of contrast-dependent center-surround antagonism in MT/V5 (Pack et al., 2005).

Our first aim was to investigate the hypothesized causal involvement of human MT/V5 in spatial suppression of motion signals. The assumption was that a disruption of neural mechanisms critically involved in spatial suppression could allow these normally suppressed motion signals to reach perceptual awareness. Specifically, we expected that disruption of MT/V5 processing would reduce spatial suppression and improve motion perception of large stimuli. By way of analogy, if one views spatial suppression as a "brake" on the processing of large motions, then the intended effect of MT/V5 disruption would effectively be equivalent to that of releasing the brake pedal. To disrupt MT/V5, 
we used offline $1 \mathrm{~Hz}$ TMS - a technique that temporarily attenuates normal functioning of the stimulated cortex with an effect that outlasts the period of stimulation (Boroojerdi et al., 2000; Battelli et al., 2009). In addition to MT/V5, we targeted early visual areas (EVA) because they also contain center-surround neurons (Jones et al., 2001; Shushruth et al., 2009) and make direct projections to MT/V5 (Born and Bradley, 2005). This additional target site is a valuable active control of nonspecific and indirect effects of TMS.

The feasibility of observing spatial suppression impairments that are evident as better-than-normal perception of large motions is supported by the existence of such observations in special populations, including the elderly (Betts et al., 2005, 2009), patients with schizophrenia (Tadin et al., 2006), and history of depression (Golomb et al., 2009). Our second aim was to determine whether TMS-dependent changes in spatial suppression might be comparable to spatial suppression abnormalities that are found in these populations, possibly yielding clues into neural correlates of observed deficits.

\section{Materials and Methods}

\section{Subjects}

We studied six subjects (ages 22-32 years, 1 female) all of whom passed TMS exclusion criteria (Rossi et al., 2009) and gave written informed consent (approved by the Beth Israel Deaconess Medical Center's Institutional Review Boards). No subject experienced any adverse effects. Subjects wore tightly fitting Lycra swimming caps. Earplugs were used to attenuate TMS noise.

\section{TMS protocol}

Selection of coil location for MT/V5 stimulation. For five subjects, we used functional magnetic resonance imaging (fMRI) MT/V5 localization and subsequent coregistration of fMRI images with each subject's head (Sack et al., 2006; Battelli et al., 2009) (supplemental Fig. 1, available at www. jneurosci.org as supplemental material); for the remaining subject, MT/V5 was localized using anatomical coordinates. Left MT/V5 was first localized using standard neuroimaging procedures (described below). These functional images were overlaid on structural brain scans and coregistered to the subject's head using Brainsight Frameless stereotaxy system (Rogue Research). Use of left MT/V5 as a target was motivated by studies demonstrating that left MT/V5 TMS is more effective at causing perceptual effects (Stewart et al., 1999; Antal et al., 2003). For all subjects, appropriate coil location was confirmed by inducing moving phosphenes using short trains of $10 \mathrm{~Hz}$ stimulation. The TMS coil was held over the marked location with its handle facing in the posterior direction at a $45^{\circ}$ angle.

Selection of coil location for occipital stimulation. The early visual cortex was localized functionally using phosphene induction (Fernandez et al., 2002). Although it is not plausible to attribute the effects of occipital TMS as exclusively targeting V1, V2 or V3, it is reasonable to assume that TMS near the occipital pole targets these early visual areas (Kastner et al., 1998). To select the stimulation location, we first marked a location in the left hemisphere $2 \mathrm{~cm}$ rostral and $2 \mathrm{~cm}$ lateral to the inion. The subjects then viewed a circle ( $8^{\circ}$ radius) at $9^{\circ}$ eccentricity in the right visual field, corresponding to the large stimulus used in the study (see Psychophysical task, below). The experimenter then adjusted the coil location until a subject reported a phosphene largely contained within the circle. The resulting location was the target for TMS of EVA, henceforth "EVA TMS." The TMS coil was held in place with its handle facing upward.

TMS stimulation. TMS was administered with a Magstim 2T Rapid stimulator (The Magstim Company Ltd) using a figure-eight $70 \mathrm{~mm}$ coil delivering biphasic pulses. Stimulation intensity was $75 \%$ of maximal stimulator output. TMS was delivered at $1 \mathrm{~Hz}$ for $15 \mathrm{~min}$ over the abovedescribed locations. Brainsight was used to continuously monitor coil position and assure consistent targeting (Gugino et al., 2001).

Previous studies have shown that $1 \mathrm{~Hz}$ TMS temporarily reduces excitability of the cortex within the stimulated area and that this effect outlasts the period of stimulation (Boroojerdi et al., 2000; Merabet et al. 2004; Allen et al., 2007). The time required to perform the belowdescribed psychophysical task $(\sim 13-16 \mathrm{~min})$ is within that for which 1 $\mathrm{Hz}$ TMS has been shown to have lasting effects in parietal regions as well as in MT/V5 (Hilgetag et al., 2001; Silvanto et al., 2008; Battelli et al., 2009). To confirm that the effect of $1 \mathrm{~Hz}$ TMS was of sufficient duration, we statistically examined trends across three trial blocks following each TMS session. The rationale was that if the effect of TMS did not outlast the experimental session, a within-session change in performance would be expected. No such trends were observed in the reported data (all $F_{(2,10)}<$ $0.74, p>0.51)$.

\section{fMRI methods}

Subjects were scanned in a 3T Phillips Intera scanner (Boston University Center for Biomedical Imaging). Standard procedures were used to localize MT/V5 (Tootell et al., 1995). Briefly, MT/V5 was localized as the region on the ascending branch of the inferior occipital sulcus that exhibited the stronger blood oxygenation level-dependent responses during blocks of expanding and contracting concentric gratings compared with the blocks of stationary concentric gratings (supplemental Fig. 1, available at www.jneurosci.org as supplemental material).

\section{Psychophysical task}

Stimuli were created in MATLAB and Psychophysics Toolbox (Brainard, 1997 ) and were shown on a linearized monitor (wide-screen 24 inch Sony GDM-FW900 CRT, $1024 \times 640$ resolution, $120 \mathrm{~Hz}$ ). Viewing was binocular at $77 \mathrm{~cm}$ and was enforced using a chin/forehead rest. Ambient and background illumination were 0.01 and $31 \mathrm{~cd} / \mathrm{m}^{2}$.

Psychophysical thresholds were measured by adaptive QUEST staircases that adjusted $\log _{10}$ (stimulus duration) and converged to $82 \%$ correct. Stimuli were drifting gratings (horizontal orientation, 1 cycle/degree, $4 \%$ s, 99\% contrast, starting phase randomized) presented in a two-dimensional raised cosine envelope, whose radius defined the stimulus size (Fig. $1 A$ ). For very brief stimuli $(\sigma<15 \mathrm{~ms})$, the temporal contrast envelope was Gaussian. Longer temporal envelopes were trapezoid-like, where flanks were halfGaussians and the central portion was set to the maximum contrast. Fine temporal precision was obtained by adjusting the SD of half-Gaussian flanks (with a constraint of $\sigma<15 \mathrm{~ms}$ ) and transferring "excess" contrast to the flat central portion. This hybrid envelope allows fine temporal precision of brief stimuli and avoids protracted fade-in/out periods associated with prolonged temporal Gaussians. Stimulus duration was defined as the width at halfheight of the temporal envelope. Subjects were instructed to fixate a central red dot.

Four stimulus conditions were used (Fig. $1 A$ ): two sizes (small, $1.2^{\circ}$ radius, or large, $8^{\circ}$ radius) at two stimulus locations $\left( \pm 9^{\circ}\right.$ horizontal eccentricity). The small stimulus size was chosen based on pilot experiments designed to estimate the optimal size at $9^{\circ}$ eccentricity (Tadin and Lappin, 2005b). The large stimulus was selected to be large enough to evoke inhibitory surround responses of MT neurons (Albright and Desimone, 1987; Raiguel et al., 1995), but small enough not to cross the vertical meridian. On each trial, a stimulus was presented at one eccentric location, with its size and location chosen pseudorandomly. Subjects indicated the perceived direction (up or down) by a key press. Feedback was provided. The next trial started $800 \mathrm{~ms}$ after subjects' responses. Thresholds were obtained in 3 successive 176-trial blocks, separated by a $10 \mathrm{~s}$ break. Each block contained 8 interleaved staircases, yielding 2 thresholds estimates for each of 4 conditions. Over 3 blocks, this yielded 6 thresholds per condition. Subjects completed all 3 blocks in 13-16 min, which ensured that all trials were completed while the effects of TMS lasted (see TMS protocol, above). Brief stimulus durations $(<100 \mathrm{~ms})$ and their unpredictable peripheral location precluded both saccadic and pursuit eye movements.

Each subject completed five sessions (Fig. $1 B$ ). The first session was conducted a day before TMS, and was used as a practice session and to generate staircase starting points for following sessions. The second session was a pre-TMS baseline. The third and fourth sessions were conducted immediately following MT/V5 and EVA TMS (order of stimulation was counterbalanced across observers). The fifth session was a post-TMS baseline. To prevent carryover effects, there was at least a 90 min break between sessions. 

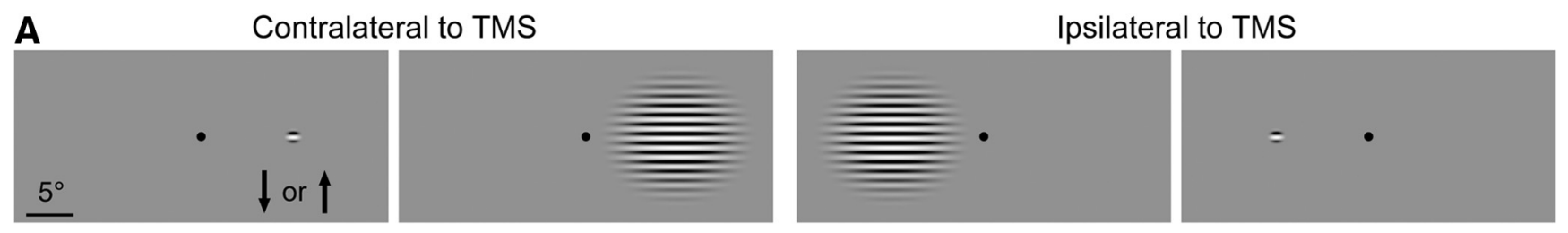

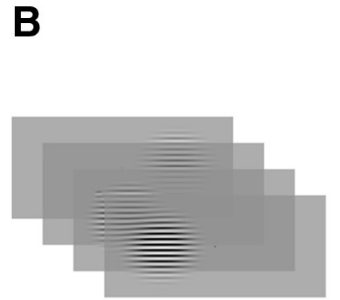

Practice

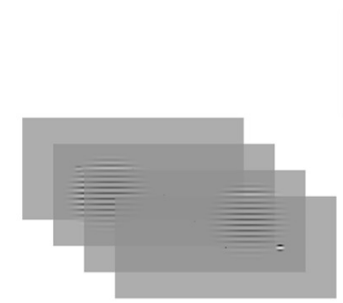

Pre-TMS

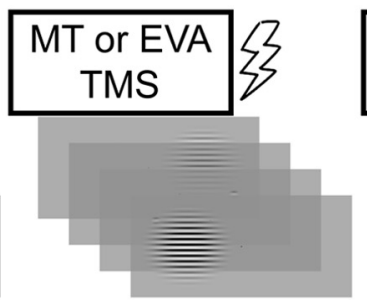

TMS session \#1

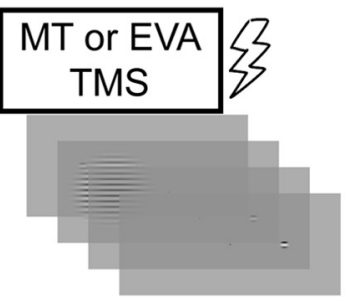

TMS session \#2

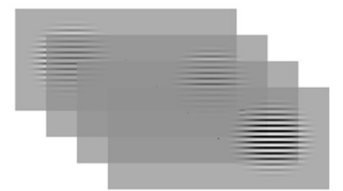

Post-TMS

Figure 1. Stimuli and procedure. $\boldsymbol{A}$, Still-frame examples of four stimulus configurations used in the study. $\boldsymbol{B}$, The timeline showing the order of five psychophysical and two TMS sessions.

\section{Results}

To measure spatial suppression strength, we used a simple task where observers discriminated motion direction of brief stimuli. Thresholds were measured for small $\left(1.2^{\circ}\right.$ radius $)$ and large $\left(8^{\circ}\right.$ radius) high-contrast stimuli that were moving either upward or downward, and presented in either the right or the left visual field (Fig. 1A). Typically, observers' direction discrimination thresholds are considerably higher for large than for small, moving stimuli-a psychophysical finding described as spatial suppression (Tadin et al., 2003). To quantify spatial suppression strength, we introduced the suppression index (SI), defined as the difference of $\log _{10}$ thresholds for large and small stimuli (Tadin et al., 2003, 2006) with higher numbers indicating stronger spatial suppression.

Results from pre-TMS baseline sessions demonstrated strong spatial suppression (left field SI $=0.41 \pm 0.04$, right field SI $=$ $0.46 \pm 0.05)$, with motion discrimination thresholds for large stimuli $(58 \pm 10 \mathrm{~ms}) \sim 3$ times higher than those for small stimuli $(20 \pm 3 \mathrm{~ms})$. These results are consistent with previous studies (Tadin et al., 2003, 2006). As no SI differences were found between pre- and post-TMS baselines (left field: $t_{5}=1.45, p=0.21$; right field: $t_{5}=0.60, p=0.58$ ), these results were averaged to create a no TMS condition. Below we present results as relative to this no TMS condition (the raw data are shown in the supplemental Fig. 2, available at www.jneurosci.org as supplemental material).

During TMS sessions, we applied 15 min of $1 \mathrm{~Hz}$ TMS to either the scalp location corresponding to left MT/V5 (supplemental Fig. 1, available at www.jneurosci.org as supplemental material) or the left occipital location where single-pulse TMS was found to elicit peripheral phosphenes (EVA TMS). Our first aim was to determine whether these experimental manipulations had an effect on spatial suppression strength. A repeatedmeasures ANOVA, with stimulus location (contralateral and ipsilateral to the stimulation) and TMS condition (no TMS, MT/V5 TMS, EVA TMS) as main factors, revealed a significant interaction $\left(F_{(2,10)}=4.0, p=0.05\right)$. Planned pairwise comparisons showed that, for contralaterally presented stimuli, spatial suppression strength was reduced following MT/V5 TMS (Fig. $\left.2 A ; t_{5}=3.72, p=0.01\right)$ but not after EVA TMS $\left(t_{5}=0.68, p=\right.$ $0.53)$. Demonstrating such specificity for the stimulated cortical area despite extensive connectivity between two stimulation sites suggests that the measurable effects of TMS are largely confined

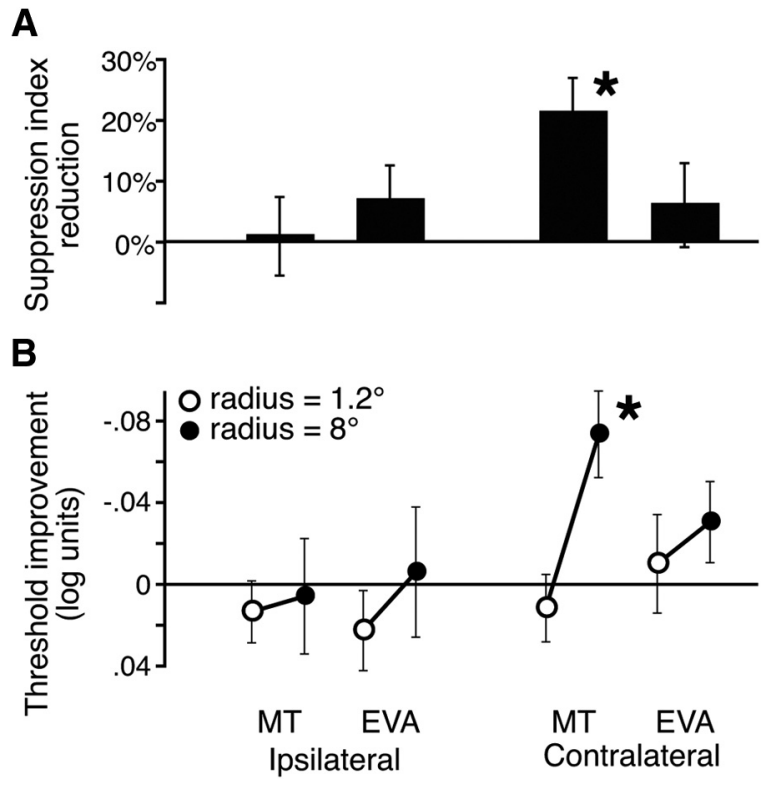

Figure 2. Effects of MT/V5 and EVA disruption. $\boldsymbol{A}, \boldsymbol{B}$, Post-TMS changes in the strength of spatial suppression $(\boldsymbol{A})$ and motion discrimination thresholds $(\boldsymbol{B})$ relative to the no TMS condition. Error bars are SEM.

to the stimulated area. For ipsilaterally presented stimuli, no SI changes were observed following MT/V5 TMS $\left(t_{5}=0.32, p=\right.$ $0.76)$, demonstrating that the underlying mechanisms exhibit at least a gross retinotopy.

The observed weakening of spatial suppression could be due to (1) increased thresholds for discriminating small, moving stimuli, (2) improved motion discriminations of large stimuli or (3) a combination of both effects. Examination of post-TMS threshold changes (Fig. $2 B$ ) showed that MT/V5 TMS had no effect on direction discriminations of small grating stimuli $\left(t_{5}=\right.$ $0.68, p=0.53$ ) (see Discussion for more details). However, as hypothesized, our results revealed improvements in motion discriminations of large, moving stimuli presented contralateral to stimulated MT/V5 $\left(t_{5}=3.45, p=0.02\right)$. Specifically, the average threshold for discriminating motion direction of large stimuli decreased by $0.072 \pm 0.02 \log$ unit, which was, on average, a 10 $\mathrm{ms}$ threshold improvement. These paradoxical results mimic special population findings; particularly patients with a history of 
depression who exhibit better-than-normal motion perception of large patterns coupled with normal perception of small, moving stimuli (Golomb et al., 2009).

It is important to consider whether these findings reflect indirect effects of TMS associated with reductions in effective stimulus speed or contrast that are known to reduce spatial suppression (Tadin et al., 2003; Lappin et al., 2009). Neither alternative, however, is consistent with our results: in addition to weakening spatial suppression, reduction of stimulus speed yields increasing duration thresholds for all sizes (Lappin et al., 2009), while a decrease in effective stimulus contrast should increase thresholds for small, moving stimuli (Tadin et al., 2003).

\section{Discussion}

Here, we report seemingly paradoxical TMS-induced improvement in motion discriminations of large, moving stimuli-a result specific to MT/V5 stimulation and the stimuli presented in the contralateral visual field. The weakening of spatial suppression during a TMS-induced period of reduced excitability of MT/V5 suggests that the critical neural constraints limiting motion perception of large, high-contrast stimuli involve visual area MT/V5. These findings are consistent with our hypothesis that behaviorally observed spatial suppression is a direct perceptual correlate of center-surround antagonism in area MT. Support for this linking hypothesis should facilitate future research efforts to elucidate functional roles of center-surround antagonism in motion perception. For example, one appealing hypothesis is that suppression of large, background-like motions facilitates rapid figure-ground segregation of moving objects by suppressing background motion signals (Born et al., 2000; Tadin and Blake, 2005).

Why does the disruption of MT/V5 attenuate behaviorally measured spatial suppression? Center-surround neurons are common in all layers of MT/V5, except in input layer IV (Raiguel et al., 1995; Born, 2000), indicating that surround inhibition in MT/V5 is not inherited from feedforward inputs. This, in turn, indicates that a selective disruption of MT/V5 processing should interfere with the development of center-surround antagonism, in turn affecting perceptual correlates of MT/V5 surround inhibition. Thus, by choosing moving stimuli large enough to activate suppressive surround of MT/V5 neurons (see Materials and Methods), we were presumably in a good position to measure any significant changes in MT/V5 surround inhibition. Although surround inhibition also occurs in a number of earlier visual areas [V1 (Jones et al., 2001); V2 (Shushruth et al., 2009)], these center-surround interactions occur at a smaller spatial scale and likely play a lesser role in suppressing neural responses to $16^{\circ}$ diameter stimuli used here.

Moreover, our selection of an experimental strategy that relies on duration thresholds and, consequentially, briefly presented stimuli ( $<100 \mathrm{~ms}$ ), likely facilitated detection of changes in surround inhibition for three specific reasons: First, considerable prior work on spatial suppression has documented the utility of this strategy (Tadin et al., 2003, 2005b, 2006; Betts et al., 2005, 2009). Second, briefly presented stimuli appear useful as selective probes of surround suppression mechanisms in MT/V5 (Churan et al., 2008). Specifically, information about motion direction of brief stimuli $(<100 \mathrm{~ms})$ in area MT/V5 is primarily carried by neurons with antagonistic surrounds, and not by MT/V5 neurons that lack suppressive surround and actually prefer large, background-like motions. Third, spatial suppression results obtained using this approach cannot be explained by sizedependent changes in contrast sensitivity (Glasser and Tadin,
2010) as might be the case for longer, counterphasing stimuli (Aaen-Stockdale et al., 2009).

One notable feature of our results is that disruption of MT/V5 yields improvements in motion perception of large stimuli coupled with unchanged motion discriminations of small gratings (see the following paragraph for a description of a similar result reported in patients with a history of depression). This finding suggests that while MT/V5 is critical for suppression of large, background-like motions, it is not solely responsible for the processing of small, first-order moving stimuli. A similar conclusion arises from lesion work, which revealed relatively unimpaired motion discriminations of grating stimuli following MT/V5 lesions in both humans (Plant and Nakayama, 1993) and nonhuman primates (Rudolph and Pasternak, 1999). The observed robustness to MT/V5 injury is likely due to widely distributed mechanisms underlying perception of first-order motion (Plant and Nakayama, 1993; Sunaert et al., 1999).

Finally, we note that our results mimic spatial suppression deficits that are observed in older adults (Betts et al., 2005, 2009), patients with schizophrenia (Tadin et al., 2006) and a history of depression (Golomb et al., 2009). Specifically, our results closely mimic Golomb et al. (2009), who also reported a weakening of spatial suppression that was due to better-than-normal perception of large, moving stimuli paired with normal perception of small, moving stimuli. It should be noted, however, that the outcome similarity between our results and patient findings does not indicate that the mechanisms of impairment are the same. While TMS is presumed to cause a gross impairment of MT/V5 functioning, special population deficits are likely more specific and probably involve a decrease in the efficacy of inhibitory mechanisms (Betts et al., 2005; Tadin et al., 2006; Golomb et al., 2009). Indeed, old age, schizophrenia, and depression have all been linked to GABAergic deficits (Leventhal et al., 2003; Wassef et al., 2003; Yoon et al., 2010). In schizophrenia, abnormal reductions of GABA concentration in visual cortex strongly correlate with abnormally weak surround suppression in the orientation domain (Yoon et al., 2010).

In summary, our results show that disruption of MT/V5 processing improves motion perception of large stimuli and, consequently, reduces the strength of spatial suppression. While the exact mechanisms underlying these changes have yet to be determined, a parsimonious conclusion is that TMS is interfering with inhibitory processing within MT/V5 that normally impairs perception of large background motions. In other words, normally functioning MT/V5 is necessary for strong spatial suppression of large moving stimuli. We speculate that this suppression of uniform background-like stimuli directly enhances saliency of smaller moving objects - a hypothesis that is a topic of our current research.

\section{References}

Aaen-Stockdale CR, Thompson B, Huang PC, Hess RF (2009) Low-level mechanisms may contribute to paradoxical motion percepts. J Vis 9:9.1-9.14.

Albright TD, Desimone R (1987) Local precision of visuotopic organization in the middle temporal area (MT) of the macaque. Exp Brain Res 65:582-592.

Allen EA, Pasley BN, Duong T, Freeman RD (2007) Transcranial magnetic stimulation elicits coupled neural and hemodynamic consequences. Science 317:1918-1921.

Antal A, Nitsche MA, Paulus W (2003) Transcranial magnetic and direct current stimulation of the visual cortex. Suppl Clin Neurophysiol 56:291-304.

Battelli L, Alvarez GA, Carlson T, Pascual-Leone A (2009) The role of the parietal lobe in visual extinction studied with transcranial magnetic stimulation. J Cogn Neurosci 21:1946-1955. 
Betts LR, Taylor CP, Sekuler AB, Bennett PJ (2005) Aging reduces centersurround antagonism in visual motion processing. Neuron 45:361-366.

Betts LR, Sekuler AB, Bennett PJ (2009) Spatial characteristics of centersurround antagonism in younger and older adults. J Vis 9:25.1-25.15.

Born RT (2000) Center-surround interactions in the middle temporal visual area of the owl monkey. J Neurophysiol 84:2658-2669.

Born RT, Bradley DC (2005) Structure and function of visual area MT. Annu Rev Neurosci 28:157-189.

Born RT, Groh JM, Zhao R, Lukasewycz SJ (2000) Segregation of object and background motion in visual area MT: effects of microstimulation on eye movements. Neuron 26:725-734.

Boroojerdi B, Prager A, Muellbacher W, Cohen LG (2000) Reduction of human visual cortex excitability using $1-\mathrm{Hz}$ transcranial magnetic stimulation. Neurology 54:1529-1531.

Brainard DH (1997) The Psychophysics Toolbox. Spat Vis 10:433-436.

Churan J, Khawaja FA, Tsui JM, Pack CC (2008) Brief motion stimuli preferentially activate surround-suppressed neurons in macaque visual area MT. Curr Biol 18:R1051-R1052.

Fernandez E, Alfaro A, Tormos JM, Climent R, Martínez M, Vilanova H, Walsh V, Pascual-Leone A (2002) Mapping of the human visual cortex using image-guided transcranial magnetic stimulation. Brain Res Brain Res Protoc 10:115-124.

Glasser DM, Tadin D (2010) Low-level mechanisms do not explain paradoxical motion percepts. J Vis 10:20.1-20.9.

Golomb JD, McDavitt JR, Ruf BM, Chen JI, Saricicek A, Maloney KH, Hu J, Chun MM, Bhagwagar Z (2009) Enhanced visual motion perception in major depressive disorder. J Neurosci 29:9072-9077.

Gugino LD, Romero JR, Aglio L, Titone D, Ramirez M, Pascual-Leone A, Grimson E, Weisenfeld N, Kikinis R, Shenton ME (2001) Transcranial magnetic stimulation coregistered with MRI: a comparison of a guided versus blind stimulation technique and its effect on evoked compound muscle action potentials. Clin Neurophysiol 112:1781-1792.

Hilgetag CC, Théoret H, Pascual-Leone A (2001) Enhanced visual spatial attention ipsilateral to rTMS-induced 'virtual lesions' of human parietal cortex. Nat Neurosci 4:953-957.

Jones HE, Grieve KL, Wang W, Sillito AM (2001) Surround suppression in primate V1. J Neurophysiol 86:2011-2028.

Kastner S, Demmer I, Ziemann U (1998) Transient visual field defects induced by transcranial magnetic stimulation over human occipital pole. Exp Brain Res 118:19-26.

Lappin JS, Tadin D, Nyquist JB, Corn AL (2009) Spatial and temporal limits of motion perception across variations in speed, eccentricity, and low vision. J Vis 9:30.1-30.14.

Leventhal AG, Wang Y, Pu M, Zhou Y, Ma Y (2003) GABA and its agonists improved visual cortical function in senescent monkeys. Science 300:812-815.

Merabet L, Thut G, Murray B, Andrews J, Hsiao S, Pascual-Leone A (2004) Feeling by sight or seeing by touch? Neuron 42:173-179.

Pack CC, Hunter JN, Born RT (2005) Contrast dependence of suppressive influences in cortical area MT of alert macaque. J Neurophysiol 93: 1809-1815.

Plant GT, Nakayama K (1993) The characteristics of residual motion perception in the hemifield contralateral to lateral occipital lesions in humans. Brain 116:1337-1353.
Raiguel S, Van Hulle MM, Xiao DK, Marcar VL, Orban GA (1995) Shape and spatial distribution of receptive fields and antagonistic motion surrounds in the middle temporal area (V5) of the macaque. Eur J Neurosci 7:2064-2082.

Rossi S, Hallett M, Rossini PM, Pascual-Leone A, Safety of TMS Consensus Group (2009) Safety, ethical considerations, and application guidelines for the use of transcranial magnetic stimulation in clinical practice and research. Clin Neurophysiol 120:2008-2039.

Rudolph K, Pasternak T (1999) Transient and permanent deficits in motion perception after lesions of cortical areas MT and MST in the macaque monkey. Cereb Cortex 9:90-100.

Sack AT, Kohler A, Linden DE, Goebel R, Muckli L (2006) The temporal characteristics of motion processing in hMT/V5+: combining fMRI and neuronavigated TMS. Neuroimage 29:1326-1335.

Schwartz O, Simoncelli EP (2001) Natural signal statistics and sensory gain control. Nat Neurosci 4:819-825.

Shushruth S, Ichida JM, Levitt JB, Angelucci A (2009) Comparison of spatial summation properties of neurons in macaque V1 and V2. J Neurophysiol 102:2069-2083.

Silvanto J, Cattaneo Z, Battelli L, Pascual-Leone A (2008) Baseline cortical excitability determines whether TMS disrupts or facilitates behavior. J Neurophysiol 99:2725-2730.

Stewart L, Battelli L, Walsh V, Cowey A (1999) Motion perception and perceptual learning studied by magnetic stimulation. Electroencephalogr Clin Neurophysiol Suppl 51:334-350.

Sunaert S, Van Hecke P, Marchal G, Orban GA (1999) Motion-responsive regions of the human brain. Exp Brain Res 127:355-370.

Tadin D, Blake R (2005) Motion perception getting better with age? Neuron 45:325-327.

Tadin D, Lappin JS (2005a) Linking psychophysics and physiology of center-surround interactions in visual motion processing. In: Seeing spatial form (Jenkin MRM, Harris LR, eds), pp 279-314. Oxford: Oxford UP.

Tadin D, Lappin JS (2005b) Optimal size for perceiving motion decreases with contrast. Vision Res 45:2059-2064.

Tadin D, Lappin JS, Gilroy LA, Blake R (2003) Perceptual consequences of centre-surround antagonism in visual motion processing. Nature 424: 312-315.

Tadin D, Kim J, Doop ML, Gibson C, Lappin JS, Blake R, Park S (2006) Weakened center-surround interactions in visual motion processing in schizophrenia. J Neurosci 26:11403-11412.

Tootell RB, Reppas JB, Kwong KK, Malach R, Born RT, Brady TJ, Rosen BR, Belliveau JW (1995) Functional analysis of human MT and related visual cortical areas using magnetic resonance imaging. J Neurosci 15: 3215-3230.

Vinje WE, Gallant JL (2000) Sparse coding and decorrelation in primary visual cortex during natural vision. Science 287:1273-1276.

Wassef A, Baker J, Kochan LD (2003) GABA and schizophrenia: a review of basic science and clinical studies. J Clin Psychopharmacol 23:601-640.

Yoon JH, Maddock RJ, Rokem A, Silver MA, Minzenberg MJ, Ragland JD, Carter CS (2010) GABA concentration is reduced in visual cortex in schizophrenia and correlates with orientation-specific surround suppression. J Neurosci 30:3777-3781. 